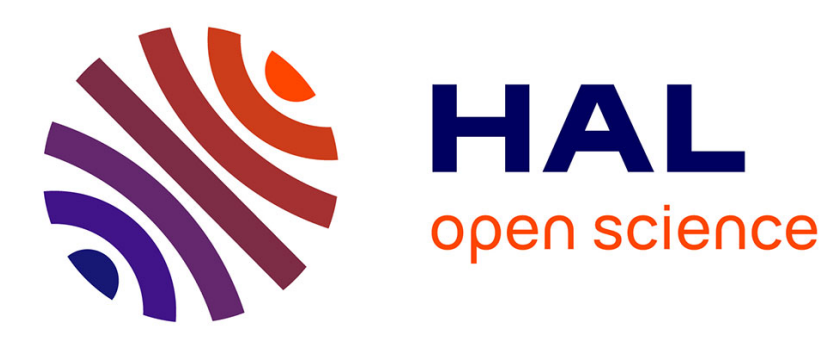

\title{
Relative hyperbolization of (one-ended hyperbolic)-by-cyclic groups
}

François Gautero, Martin Lustig

\section{To cite this version:}

François Gautero, Martin Lustig. Relative hyperbolization of (one-ended hyperbolic)-by-cyclic groups. Mathematical Proceedings of the Cambridge Philosophical Society, 2004, 137 (3), pp.595-611. hal00808792

\section{HAL Id: hal-00808792 \\ https://hal.science/hal-00808792}

Submitted on 7 Apr 2013

HAL is a multi-disciplinary open access archive for the deposit and dissemination of scientific research documents, whether they are published or not. The documents may come from teaching and research institutions in France or abroad, or from public or private research centers.
L'archive ouverte pluridisciplinaire HAL, est destinée au dépôt et à la diffusion de documents scientifiques de niveau recherche, publiés ou non, émanant des établissements d'enseignement et de recherche français ou étrangers, des laboratoires publics ou privés. 


\title{
Relative hyperbolization of (one-ended hyperbolic)-by-cyclic groups
}

\author{
François Gautero and Martin Lustig
}

\begin{abstract}
We show that the semi-direct product of a one-ended torsion-free wordhyperbolic group with $\mathbb{Z}$, given through an automorphism $\alpha: G \rightarrow G$, is a hyperbolic group relative to certain canonical subgroups of $G$ on which $\alpha$ acts periodically or with linear growth.
\end{abstract}

\section{Introduction}

Let $G$ be a word-hyperbolic group in the sense of Gromov [17], and let $\alpha$ be an automorphism of $G$. For simplicity we consider in this paper only the case of torsion-free $G$. The overall question adressed in this paper is whether (or better, to what extent) the mapping torus group of $\alpha$, i.e. the semi-direct product

$$
G_{\alpha}=G \rtimes_{\alpha} \mathbb{Z}
$$

is itself a word-hyperbolic group. The general answer to this question is mixed, depending on the properties of the automorphism $\alpha$. The best way to phrase this is in terms of relative hyperbolicity (as introduced by Farb), explained in detail in section 6 .

Theorem 0.1. For any freely indecomposable non-elementary torsion-free wordhyperbolic group $G$ and every automorphism $\alpha$ of $G$ the semi-direct product $G \rtimes_{\alpha} \mathbb{Z}$ is hyperbolic relative to a family of maximal subgroups of $G$ which consist entirely of elements on which $\alpha$ acts up to conjugacy periodically or with linear growth.

Notice that the statement of Theorem 0.1 is sharp in the sense that it becomes wrong whenever one tries to decrease the relative part essentially: Indeed, every element of an $\alpha$-periodic conjugacy class in $G$ is contained in a $\mathbb{Z} \oplus \mathbb{Z}$ subgroup of $G_{\alpha}$. A similar statement is true for a linearily growing element, relative to the family of periodic subgroups. For any $\mathbb{Z} \oplus \mathbb{Z}$ subgroup, one of its summands must lie (up to conjugacy) in the relative part in order to get a relatively hyperbolic group. These subgroups also explain why above we use Farb's version of relative hyperbolicity: In order for $G_{\alpha}$ to be relatively hyperbolic in the stronger sense of Gromov, both factors of any $\mathbb{Z} \oplus \mathbb{Z}$ subgroup must be contained in the relative part. Such a coarser "factorization" of $G_{\alpha}$, with a stronger relative hyperbolicity property, will be discussed in Remark 7.4. 
Remark 0.2. If $G$ is a non-trivial free product, then its automorphisms behave, relative to the free factors, very much like automorphisms of free groups (compare [7]), so that the results of [13] can be adapted. In combination with the results and methods of the present paper, with a small amount of extra technical work, [13] gives the prospect of a complete solution of our overall question for all automorphisms $\alpha$ of all (torsion-free) hyperbolic groups $G$, stated as follows: $G_{\alpha}=G \rtimes_{\alpha} \mathbb{Z}$ is hyperbolic relative to a canonical set of subgroups of $G$ that consist entirely of (and contain up to conjugation all) elements that have polynomial $\alpha$-growth.

Using Sela's decomposition of one-ended hyperbolic groups, described in section 7 below, the above Theorem 0.1 will follow directly from the more general statement of Theorem 7.1, where $G$ is replaced by the fundamental group of a graph of groups of a certain type.

An important special case, which is at the time a "warm up" as well as the most important building block in the proof of these results, is that of a surface group $G$, where $\alpha$ is given by a surface homeomorphism.

Theorem 0.3. Let $M_{h}$ be the mapping cylinder of a homeomorphism $h: S \rightarrow S$ of a surface $S$ with Euler characterisitc $\chi(S)<0$, possibly with non-empty boundary $\partial S$. Then the fundamental group $\pi_{1} M_{h}$ is hyperbolic relative to the family of subgroups defined (up to conjugation) by the fundamental groups of the boundary curves of $S$, of the reduction curves in the Nielsen-Thurston reduction of $h$, and of those subsurfaces of $S$ which are maximal unions of components in this reduction on which $h$ induces a periodic mapping class, pasted together along a maximal subfamily of the reduction curves.

Before attacking the case of reducible $h$ in section 6 we very carefully consider the "absolute" case of a closed surface $S$ provided with a pseudo-Anosov homeomorphism $h: S \rightarrow S$. (Note that, if the mapping class of $h$ is reducible or of finite order, then the mapping-torus $M_{h}$ of $h$ contains a $\pi_{1}$-injective 2-torus, and hence its fundamental group contains a copy of $\mathbb{Z} \oplus \mathbb{Z}$ and thus can not be wordhyperbolic.) The first five sections of this paper are devoted to a detailed analysis of the geometry of mapping tori of such pseudo-Anosov surface homeomorphisms. We assemble the tools necessary for the more difficult relative case, and, as a side product, we also obtain a conceptually simple proof of the following:

Proposition 0.4. If the mapping class of a surface homeomorphism $h: S \rightarrow S$ is pseudo-Anosov, then $\pi_{1} M_{h}$ is word-hyperbolic.

Note that Proposition 0.4 is also a well known consequence of each of three important, highly non-trivial theories: of Thurston's hyperbolisation of fibered 3-manifolds [19], of Bestvina-Feighn's combination theorem [1], or of the work of Gabai-Kazez [12],[11] on genuine essential laminations in 3-manifolds.

By contrast, the methods presented in this paper only use classical NielsenThurston surface theory [10] and elementary geometric considerations: We use the stable and the unstable singular foliation on $S$, which is associated to the 
pseudo-Anosov map $h$, to define a foliation metric on the universal cover of $S$. This foliation metric is lifted and properly rescaled to define a metric on the universal cover $\tilde{M}$ of $M_{h}$ (reminiscent of the metric used by Cannon-Thurston, see $[5,8])$. This metric is preserved by the free, properly discontinuous and cocompact action of $\pi_{1} M_{h}$. Hence, in order to prove that $\pi_{1} M_{h}$ is hyperbolic, it suffices to show that geodesic triangles in $\tilde{M}$ are thin (see [4] and [15] for background). This is done through a close investigation of our metric on $\tilde{M}$, which results in an approximation of geodesics by vertical and horizontal segments. We find this geodesic approximation illuminating in its own way, also with respect to potential further applications.

Acknowledgements : The first author would like to thank the Mathematics Department of University of Geneva where he held a post-doctoral position while most of this work was done.

\section{Setting}

From here until the end of section 5 we consider a compact, connected surface $S$ with empty boundary and with Euler characteristic $\chi(S)<0$, as well as a pseudo-Anosov homeomorphism $h$ of $S$. The surface $S$ can be endowed with a hyperbolic structure, given by an identification of the universal cover of $S$ with the Poincaré disk $D^{2}$. The action of the fundamental group $\pi_{1} S$ on the universal cover of $S$ by deck transformations is realized via this identification by isometries on $D^{2}$ with respect to Poincaré's hyperbolic metric.

We choose a lift $\tilde{h}$ of $h$ to $D^{2}$. It admits a pair of $\pi_{1} S$-equivariant, $\tilde{h}$-invariant, transverse, singular foliations $\mathcal{F}_{u}, \mathcal{F}_{s}$ in $D^{2}$, called the unstable and the stable foliation respectively. Both $\mathcal{F}_{u}$ and $\mathcal{F}_{s}$ come with $\pi_{1} S$-equivariant, transverse measures, denoted $\mu_{u}$ and $\mu_{s}$. The transverse measure $\mu_{s}$ on $\mathcal{F}_{s}$ is uniformly dilated by a factor $\lambda>1$ under the induced action of $\tilde{h}$, and similarly $\mu_{u}$ is uniformly contracted by the factor $\frac{1}{\lambda}$. In particular this gives for any finite segment $l_{0}$ of a leaf $l$ of $\mathcal{F}_{s}$, and for any finite segment $l_{0}^{\prime}$ of a leaf $l^{\prime}$ of $\mathcal{F}_{u}$, the equations (compare [10]):

$$
\mu_{u}\left(\tilde{h}\left(l_{0}\right)\right)=\frac{1}{\lambda} \mu_{u}\left(l_{0}\right), \quad \mu_{s}\left(\tilde{h}\left(l_{0}^{\prime}\right)\right)=\lambda \mu_{s}\left(l_{0}^{\prime}\right)
$$

The universal cover of the mapping torus $M_{h}=S \times[0,1] /\{(s, 1)=(h(s), 0) \mid s \in$ $S\}$ is called mapping telescope and denoted by $\tilde{M}$. It is homeomorphic to $D^{2} \times \mathbb{R}$, but for our purposes we have to carefully select the identification $\tilde{M}=D^{2} \times \mathbb{R}$ among the different (natural) possibilities. This is most conveniently done by specifying a free, properly discontinuous and cocompact action of $\pi_{1} M_{h}$ on $D^{2} \times \mathbb{R}$, and by identifying $M_{h}$ with the quotient space:

Every element $g \tau^{r}$ of $\pi_{1} M_{h}=\pi_{1} S \rtimes_{\tilde{h}_{*}} \mathbb{Z}$, for $g \in \pi_{1} S, \tau$ a generator of the infinite cyclic "right factor" $\mathbb{Z}$ and $r$ any integer, can be identified with the lift $H=g \tilde{h}^{r}: D^{2} \rightarrow D^{2}$ of the $r$-th power of the given homeomorphism $h: S \rightarrow S$. 
We define the corresponding "deck transformation" $\hat{H}$ on $\tilde{M}$ by

$$
\hat{H}\left(g \tau^{r}\right)=\hat{H}: D^{2} \times \mathbb{R} \rightarrow D^{2} \times \mathbb{R},(x, t) \mapsto(H(x), t-r) .
$$

For $r=0$ this gives the (desired) consequence that an element $g \in \pi_{1} S$ acts on $\tilde{M}=D^{2} \times \mathbb{R}$ through $g(x, t)=(g x, t)$. In particular, the quotient manifold $\left(D^{2} \times\right.$ $\mathbb{R}) / \pi_{1} M_{h}$ is obtained in two steps as follows: First one quotients $D^{2} \times \mathbb{R}$ modulo $\pi_{1} S$ to obtain $S \times \mathbb{R}$, on which the infinite cyclic group $\pi_{1} M_{h} / \pi_{1} S$ generated by the image $\bar{\tau}$ of $\tau$ acts through $\bar{\tau}(z, t)=(h(z), t-1)$. As second step one quotients $S \times \mathbb{R}$ modulo $<\bar{\tau}>$, which clearly gives the mapping torus $M_{h}$.

As a consequence, for each time $t$ the fibre $F_{t}=D^{2} \times\{t\}$ of $\tilde{M}$ covers a fibre homeomorphic to $S$ of the mapping torus fibration $M_{h} \rightarrow S^{1}$. More specifically, the covering map $D^{2} \times \mathbb{R} \rightarrow M_{h}$ induces for every integer value $t \in \mathbb{Z} \subset \mathbb{R}$ a covering map $D^{2} \times\{t\} \rightarrow S,(x, t) \mapsto y$ which differs from the above specified universal covering map $D^{2} \rightarrow S, x \mapsto z$ by composition with the power $h^{t}$, i.e. $y=h^{-t}(z)$. The analogous statement is true for any two fibres $F_{t_{1}}, F_{t_{2}}$ at times $t_{1}, t_{2}=t_{1}+t$ which differ by an integer $t \in \mathbb{Z}$.

We now rescale the transverse measures on the fibres continuously, by multiplying $\mu_{u}$ with the factor $\lambda^{-t}$ to get the transverse measure of the unstable foliation on $D^{2} \times\{t\}$, and by multiplying $\mu_{s}$ with factor $\lambda^{t}$ to get the transverse measure of the unstable foliation on $D^{2} \times\{t\}$. This has the effect that any of the above defined deck transformations $\hat{H}: D^{2} \times \mathbb{R} \rightarrow D^{2} \times \mathbb{R}$ preserves both transverse measures of any path which is contained in some fibre $F_{t} \subset \tilde{M}$.

We define the telescope flow $\Phi_{t}: \tilde{M} \rightarrow \tilde{M}$ by $\Phi_{t}(x, r)=(x, r+t)$. This is nothing other than the lift under the covering map of the mapping torus flow on $M_{h}$. If $P=(x, t)$ is a point in $\tilde{M}$, we will denote by $O_{P}=\{x\} \times \mathbb{R}$ its orbit under the telescope flow. Note that the above rescaling of the metric has the effect that two orbits $O_{P}, O_{Q}$ in the same stable leaf will approach one another arbitrarily close and exponentially fast in the future (i.e. for $t>0$ ), and they will diverge exponentially fast in the past. The converse is true for unstable leaves.

\section{The foliation metric on the Poincaré disk}

In the Poincaré disk $D^{2}$, identified above with the universal cover of the surface $S$, we use the two foliations $\mathcal{F}_{u}, \mathcal{F}_{s}$ and their transverse measures $\mu_{u}, \mu_{s}$ to define a new metric:

The unstable length $|\gamma|_{u}$ (resp. stable length $|\gamma|_{s}$ ) of a path $\gamma$ in $D^{2}$ is the total variation of $\left|d \mu_{u}\right|$ (resp. $\left.\left|d \mu_{s}\right|\right)$ along $\gamma$. The foliation distance $d_{\mathcal{F}}(x, y)$ between any two points $x, y$ in $D^{2}$ is the infimum, over all the paths $\gamma$ between $x$ and $y$, of the foliation length $|\gamma|_{\mathcal{F}}=|\gamma|_{u}+|\gamma|_{s}$.

We denote by $D_{\mathcal{F}}^{2}$ the Poincaré disk equipped with the foliation distance $d_{\mathcal{F}}$. The latter is clearly equivariant with respect to the covering space action of $\pi_{1} S$. As this action is free, properly discontinuous and cocompact, the disk $D_{\mathcal{F}}^{2}$ is quasi-isometric to the group $\pi_{1} M_{h}$. In particular one has:

Proposition 2.1. The disk $D_{\mathcal{F}}^{2}$ is a Gromov hyperbolic metric space. 
Similarly, each fibre $F_{t}=D^{2} \times\{t\}$ of $\tilde{M}$ can be equipped with the analogous foliation metric, denoted below by $d_{\mathcal{F}}^{t}$. The unstable and the stable foliation length in $F_{t}$ will be denoted by $|\gamma|_{u}^{t}$ and $|\gamma|_{s}^{t}$ respectively. Since $F_{t+1}$ is isometric to $F_{t}$ (through the deck transformation $\hat{H}$ associated in section 1 to any lift $H$ of the homeomorphism $h: S \rightarrow S$ ), and since the Gromov hyperbolicity constant $\delta_{t}$ for $F_{t}$ varies continuously with $t \in \mathbb{R}$, there is an upper bound $\delta>0$ to all $\delta_{t}$.

The following is an elementary exercise (which becomes almost trivial, if one passes from measured foliations to the associated geodesic laminations).

Lemma 2.2. (i) A path $\gamma:[0, d] \rightarrow D_{\mathcal{F}}^{2}$ is a geodesic if and only if it intersects any leaf of $\mathcal{F}_{u} \cup \mathcal{F}_{s}$ in such a fashion that the full $\gamma$-preimage of the intersection is connected.

(ii) A geodesic $\gamma$ intersects each leaf of $\mathcal{F}_{u} \cup \mathcal{F}_{s}$ which separates the endpoints of $\gamma$, and no other leaf.

(iii) Any two geodesics in $D_{\mathcal{F}}^{2}$ with same endpoints have the same unstable and the same stable length.

Lemma 2.3. Let $l$ be a leaf of $\mathcal{F}_{u}$ which separates two points $A, B \in D_{\mathcal{F}}^{2}$, and let $l^{\prime}$ be a similar leaf of $\mathcal{F}_{s}$. If $l$ and $l^{\prime}$ intersect, then the intersection point $Y \in D_{\mathcal{F}}^{2}$ is contained in some geodesic between $A$ and $B$. In particular, any two geodesics $[A, Y]$ and $[Y, B]$ intersect only in $Y$, and their union is a geodesic $[A, B]$.

Proof. Assume $Y_{0}$ is a point on both $[A, Y]$ and $[Y, B]$, and let $l_{0}$ be the leaf of $\mathcal{F}_{u}$ and $l_{0}^{\prime}$ the leaf of $\mathcal{F}_{s}$ that contain $Y_{0}$. If $Y_{0} \neq Y$, then by Lemma 2.2 (ii) $l_{0}$ or $l_{0}^{\prime}$ separates $Y$ from both $A$ and $B$. But then one of the leaves $l$ or $l^{\prime}$ that contain $Y$ must also be separated by $l_{0}$ or $l_{0}^{\prime}$ from both $A$ and $B$. Hence one of them does not separate $A$ from $B$, which contradicts the assumption on $l$ or that on $l^{\prime}$.

Proposition 2.4. Let $A, B, C \in D_{\mathcal{F}}^{2}$ be arbitrary three points. Then there exists a point $Z \in D_{\mathcal{F}}^{2}$ which lies on three properly chosen geodesics $[A, B],[B, C]$ and $[A, C]$. In particular there is a geodesic triangle $\Delta=[A, B, C]$ which is a tripod with centre $Z$.

Proof. Any leaf of either $\mathcal{F}_{u}$ or $\mathcal{F}_{s}$ which intersects one side of a geodesic triangle $[A, B, C]$ must also intersect at least one other side. One observes that there must be precisely one special leaf in either foliation which intersects all three sides of $[A, B, C]$. The special leaf can be regular, in which case it must contain at least one vertex of the triangle. According to the location of the intersection points of the two special leaves from $\mathcal{F}_{u}$ and $\mathcal{F}_{s}$ with the sides of $[A, B, C]$ one distinguishes several cases, and for each of them one sees directly that the two special leaves must intersect. Hence we can apply Lemma 2.3 to derive that the intersection point $Z$ of the two special leaves has all the desired properties.

From the arguments in the last proof it follows also that the centre $Z$ is uniquely determined by the three given points $A, B, C$, but this fact is not used here. 


\section{The telescope distance on $\tilde{M}$}

For any two points $P, Q \in \tilde{M}$ and for any time $t \in \mathbb{R}$ let $P_{t} \in O_{P}$ and $Q_{t} \in O_{Q}$ be the intersection points of the fibre $F_{t}$ with the orbits $O_{P}$ and $O_{Q}$ respectively. From the definition of the foliation metric in $F_{t}$ in the last section it follows directly that, unless $P_{t}$ and $Q_{t}$ belong to a leaf of $\mathcal{F}_{s}$ or of $\mathcal{F}_{u}$, the distance $d_{\mathcal{F}}^{t}\left(P_{t}, Q_{t}\right)$ becomes minimal at the diagonal time $t_{\text {diag }}$ for $P$ and $Q$, by which we mean the time coordinate $t=t_{\text {diag }}$ of the fibre $F_{t}$ where the stable and the unstable distances between $P_{t}$ and $Q_{t}$ are equal. This distance is called the orbit width of $O_{P}$ and $O_{Q}$ and denoted by $\omega(P, Q)$. The points $P_{t_{\text {diag }}}$ and $Q_{t_{\text {diag }}}$ are said to lie in diagonal position. Away from the diagonal fibre $F_{t_{\text {diag }}}$ the distance $d_{\mathcal{F}}^{t}\left(P_{t}, Q_{t}\right)$ increases with $t$ into both directions, in an exponential (or more precisely, a hyperbolic cosine like) fashion.

If $P_{t}$ and $Q_{t}$ are contained in the same leaf of $\mathcal{F}_{s}$ or of $\mathcal{F}_{u}$, we set $\omega(P, Q)=0$ and $t_{\text {diag }}=\infty$ or $t_{\text {diag }}=-\infty$.

We say that two points $P^{\prime}, R^{\prime}$ in some fibre $F_{t}$ are in $(\epsilon, \delta)$-almost diagonal position, for $\epsilon, \delta \geq 0$, if there are points $P$ and $R$ in the $\delta$-neighbourhood of $P^{\prime}$ and $R^{\prime}$ respectively, which are in diagonal position and which satisfy $d_{\mathcal{F}}^{t}(P, R) \geq \epsilon$. We observe:

Lemma 3.1. For any sufficiently large $\epsilon>2 \delta$ there exists a constant $\sigma>0$ such that for any two points $P^{\prime}, R^{\prime}$ in some fibre $F_{t}$ which are in $(\epsilon, \delta)$-almost diagonal position, the diagonal time $t^{\prime}$ for $P^{\prime}, R^{\prime}$ satisfies $\left|t-t^{\prime}\right| \leq \sigma$.

A path $\gamma$ in $\tilde{M}$ is called vertizontal if it is the concatenation of finitely many subpaths that are vertical (i.e. segments of some orbit $O_{P}$ ) or horizontal (i.e. contained in some fibre $\left.F_{t}\right)$. The length $l(\gamma)$ of such a path is simply the sum of the length of their subpaths, where each horizontal subpath $\gamma^{\prime} \subset F_{t}$ is measured in the foliation metric $d_{\mathcal{F}}^{t}$ defined on $F_{t}$, while the length of a vertical segment is simply the difference of the time coordinates of its endpoints.

Lemma 3.2. Let $\gamma$ be a vertizontal path in $\tilde{M}$ from $P=\left(x_{P}, t_{P}\right)$ to $R=\left(x_{R}, t_{R}\right)$, and assume that $\gamma$ realizes at the point $Q_{\min }=\left(x_{\text {min }}, t_{\text {min }}\right)$ the minimal time coordinate. Let $\gamma_{\min }$ be the projection of $\gamma$ under the telescope flow to the fibre $F_{t_{\text {min }}}$. Then the length of $\gamma$ satisfies the inequality:

$$
l(\gamma) \geq\left|\gamma_{\min }\right|_{u}^{t_{\min }}+t_{P}+t_{R}-2 t_{\min }
$$

Similarly, if $Q_{\max }=\left(x_{\max }, t_{\max }\right)$ is the point of $\gamma$ with the maximal time coordinate, then one has, for the projection $\gamma_{\max } \subset F_{t_{\max }}$ of $\gamma$ :

$$
l(\gamma) \geq\left|\gamma_{\max }\right|_{s}^{t_{\max }}-t_{P}-t_{R}+2 t_{\max }
$$

Proof. Between $P$ and $Q_{\text {min }}$ the path $\gamma$ accumulates a time contribution of at least $t_{P}-t_{\min }$, and between $Q_{\min }$ and $R$ it accumulates similarly at least $t_{R}-t_{\text {min }}$. From the scaling of the foliation metric in the fibres according to time we obtain that the unstable contribution to $l(\gamma)$ is bounded below by 
$\left|\gamma_{\min }\right|_{u}^{t_{\min }}$, which proves the first inequality. The second one is completely analogous.

We define a metric $d$ on $\tilde{M}$ (the telescope metric) as follows:

Definition 3.3. For any two points $P, Q \in \tilde{M}$ let $d(P, Q)$ denote the infimum of the lengths $l(\gamma)$ of all vertizontal paths $\gamma$ joining $P$ to $Q$.

Using a decomposition of $S$ into rectangles with sides given by stable and unstable leaves (as done in the usual Markov partitions for $h: S \rightarrow S$, see [10]) it is not hard to see that this infimum is indeed realized by some vertizontal path, which hence is a geodesic $[P, Q]$. Thus $\tilde{M}$ is a proper geodesic metric space on which $\pi_{1} M_{h}$ acts by isometries in a free, cocompact and properly discontinuous way, so that $\tilde{M}$ and $\pi_{1} M_{h}$ are quasi-isometric. In particular, in order to show that $\pi_{1} M_{h}$ is word-hyperbolic, it suffices to show that geodesic triangles in $\tilde{M}$ are thin.

Lemma 3.4. The image $\gamma_{t} \subset F_{t}$ of a geodesic $\gamma \subset \tilde{M}$ under the telescope flow is a geodesic with respect to the foliation metric $d_{\mathcal{F}}^{t}$.

Proof. If $\gamma_{t}$ is not a geodesic, then by Lemma 2.2 (ii) it intersects some leaf $l^{\prime}$ of the stable or the unstable foliation which does not separate the endpoints of $\gamma_{t}$. In particular, $\gamma_{t}$ intersects twice another leaf $l$ that separates the endpoints of $\gamma_{t}$ from $l^{\prime}$. Hence we can strictly reduce the length of $\gamma$ by "shortcutting" a subpath $\gamma^{\prime}$ with endpoints in the full lift $L \subset \tilde{M}$ of $l$, and $\gamma^{\prime}$ intersects the full lift of $l^{\prime}$ : We replace $\gamma^{\prime}$ with a path $\gamma^{\prime \prime}$ that is entirely contained in $L$. Here $\gamma^{\prime \prime}$ is the projection of $\gamma^{\prime}$ into $L$ along leaves of the stable or the unstable foliations in the fibres $F_{t}$ which intersect $\gamma^{\prime}$.

A path $\gamma=[A, B] \subset \tilde{M}$ from $A$ to $B$ is called a geodesic lift (of $\gamma_{t}$ ), if the telescope flow projection $\gamma_{t}$ of $\gamma$ into any fibres $F_{t}$ is a geodesic in $F_{t}$ with respect to the metric $d_{\mathcal{F}}^{t}$, and if there is no strictly shorter path in $\tilde{M}$ from $A$ to $B$ which also projects to $\gamma_{t}$. Clearly every geodesic in $\tilde{M}$ is a geodesic lift. Similarly as shown above for geodesics one can see that a vertizontal geodesic lift exists for any $d_{\mathcal{F}}^{t}$-geodesic in a fibre $F_{t}$.

\section{Geodesics in $\tilde{M}$}

The goal of this section is to show that any geodesic in $\tilde{M}$ locally either has an "almost vertical" direction, in which case it stays close to an orbit $O_{X}$, or else, if it has a "mostly horizontal" direction, it passes close to the diagonal projection of a properly chosen subsegment of the geodesic. The precise statement is given by the following three propositions.

For any $c>0$ we say that a subset $S$ of $\tilde{M}$ is a $c$-approximation of a subset $T \subset \tilde{M}$, if $S$ and $T$ are contained in the $c$-neighbourhood of each other (i.e. if their Hausdorff distance is smaller or equal to $c$ ). We say that a point $Q \in \tilde{M}$ is 
contained in the horizontal c-neighbourhood of an orbit $O_{P}$ if in the fibre $F_{Q}$ that contains $Q$ there is a path $\gamma_{Q}$ which connects $Q$ to $P^{\prime}=O_{P} \cap F_{Q}$ and is of length $l\left(\gamma_{Q}\right) \leq c$.

Proposition 4.1. For any $d>0$ there exists a bound $c=c(d)>0$ with the following property, for any points $X, Y \in \tilde{M}$ :

If $Y$ is contained in the horizontal d-neighbourhood of the orbit $O_{X}$, then any geodesic lift $\gamma=[X, Y]$ is contained in the horizontal c-neighbourhood of $O_{X}$. Furthermore, $\gamma$ is c-approximated by the vertical segment $\left[X, X^{\prime}\right] \subset O_{X}$, where $X^{\prime} \in O_{X}$ is the point in the same fibre $F_{Y}$ as $Y$.

Proof. For any point $Z \in \gamma$ let $t_{Z}$ denote the time coordinate of $Z$. We consider the projection $\gamma_{Z}$ of the initial segment $[X, Z]$ of $\gamma$ into the fibre $F_{Z}$ that contains $Z$. It suffices to show that $t_{Z}$ lies in a neighbourhood of $\left[t, t_{Y}\right] \subset \mathbb{R}$ which depends only on $d$, and that both the stable and the unstable length of $\gamma_{Z}$ are bounded above by a constant which also depends only on $d$.

We consider the vertizontal path $\gamma^{\prime}$ which is the union of the vertical segment $\left[X, X^{\prime}\right]$ with the projection $\gamma_{Y} \subset F_{Y}$ of $\gamma$, and observe:

$$
l(\gamma) \leq l\left(\gamma^{\prime}\right) \leq\left|t_{X}-t_{Y}\right|+d
$$

By symmetry we can assume that $t_{X} \geq t_{Y}$, so that the above estimate shows that $\gamma$ must be contained between the fibres $F_{t_{Y}-\frac{d}{2}}$ and $F_{t_{X}+\frac{d}{2}}$. This establishes the desired bounds for $t_{Z}$. We also deduce, for any point $Z \in \gamma$, that the stable length of $\gamma_{Z}$ is bounded above by the stable length of the projection $\gamma_{t_{Y}-\frac{d}{2}}$ of $\gamma$ to $F_{t_{Y}-\frac{d}{2}}$ which depends only on $d$ :

$$
\left|\gamma_{Z}\right|_{s}^{t_{Z}} \leq\left|\gamma_{t_{Y}-\frac{d}{2}}\right|_{s}^{t_{Y}-\frac{d}{2}} \leq \lambda^{\frac{d}{2}} d
$$

To get the bound on the unstable length of $\gamma_{Z}$ we apply Lemma 3.2 with $P=Z$ and $R=X$ and obtain, for the smallest time coordinate $t_{\min }$ of any point $Q_{\min } \in$ $[X, Z]$, and for the projection $\gamma_{\min }$ of $[X, Z]$ to the fibre $F_{t_{m i n}}$, the inequality

$$
l([X, Z]) \geq\left|\gamma_{\min }\right|_{u}^{t_{\min }}+t_{X}+t_{Z}-2 t_{\min }
$$

and thus

$$
\begin{gathered}
\left(t_{Z}-t_{Y}\right)+\left(\left|\gamma_{\min }\right|_{u}^{t_{\min }}+t_{X}+t_{Z}-2 t_{\min }\right) \leq l([Y, Z])+l([Z, X])= \\
l(\gamma) \leq l\left(\gamma^{\prime}\right) \leq t_{X}-t_{Y}+d,
\end{gathered}
$$

which gives

$$
\left|\gamma_{\min }\right|_{u}^{t_{\min }}+2\left(t_{Z}-t_{\min }\right) \leq d
$$

From the last inequality we deduce directly the desired upper bound

$$
\left|\gamma_{Z}\right|_{u}^{t_{Z}}=\lambda^{t_{Z}-t_{\min }}\left|\gamma_{\min }\right|_{u}^{t_{\min }} \leq \lambda^{\frac{d}{2}} d
$$

which depends only on $d$. 
Lemma 4.2. (a) For any $h>0$ there exists $j=j(h)>0$ with the following property: Let $\gamma=[P, R]$ be any geodesic lift in $\tilde{M}$ that joins orbits of width $\omega(P, R) \leq h$, and let $F_{t}$ be the fibre closest to $P$ with the property that the projection $\gamma_{t} \subset F_{t}$ of $\gamma$ has length $l\left(\gamma_{t}\right) \leq h$. Then $\gamma$ passes through the $j$-neighbourhood of $\gamma_{t}$.

(b) For any $0<h_{0} \leq h_{1}$ there exists a bound $j\left(h_{0}, h_{1}\right)>0$ such that for any $h$ with $h_{0} \leq h \leq h_{1}$ one has $j(h) \leq j\left(h_{0}, h_{1}\right)$.

Proof. (a) If the endpoints of $\gamma$ lie on different sides of $F_{t}$, then the claim is obvious, as there is a point where $\gamma$ has to meet $F_{t}$ and hence $\gamma_{t}$. Hence we can assume that $\gamma$ lies completely on one side of $F_{t}$, and by symmetry we can assume that $t<t_{\text {min }}$, where we use the same notation as in Lemma 3.2. The latter gives $l(\gamma) \geq\left|\gamma_{\min }\right|_{u}^{t_{\min }}+t_{P}+t_{R}-2 t_{\min }$. Note that the assumption $t<t_{\min }$ implies also that $t \geq t_{\text {diag }}$, where $t_{\text {diag }}$ denotes the time coordinate of the diagonal projection of $\gamma$, so that we deduce that the unstable length of $\gamma_{t}$ is bigger than the stable one and hence also bigger than $\frac{h}{2}$.

Now we consider the vertizontal path $\gamma^{\prime}$ which descends from the endpoint $P$ of $\gamma$ along the orbit $O_{P}$ down to $F_{t}$, then proceeds along the projection $\gamma_{t}$, and finally reconnects to the other endpoint $R$ of $\gamma$ along the orbit $O_{R}$. The length of $\gamma^{\prime}$ is equal to $l\left(\gamma^{\prime}\right)=t_{P}-t+t_{R}-t+l\left(\gamma_{t}\right) \leq t_{P}-t+t_{R}-t+h$, which has to be greater or equal to the length of the geodesic lift $\gamma$. Hence we obtain $\left|\gamma_{\text {min }}\right|_{u}^{t_{\text {min }}}+t_{P}+t_{R}-2 t_{\text {min }} \leq l(\gamma) \leq l\left(\gamma^{\prime}\right) \leq t_{P}-t+t_{R}-t+h$, thus $2\left(t_{\text {min }}-t\right)+h \geq\left|\gamma_{\text {min }}\right|_{u}^{t_{\text {min }}} \geq \lambda^{t_{\text {min }}-t}\left|\gamma_{t}\right|_{u}^{t} \geq \lambda^{t_{\text {min }}-t} \frac{h}{2}$, and finally

$$
4\left(t_{\text {min }}-t\right)+\left(2-\lambda^{t_{\text {min }}-t}\right) h \geq 0
$$

which implies that $t_{\min }-t$ is bounded above by some $j>0$ which only depends on $h$.

(b) The statement (b) is a direct consequence of the last inequality.

Notice that in the previous lemma the fibre $F_{t}$ can not be replaced by the diagonal fibre for the orbits $O_{P}$ and $O_{R}$ : In fact, for small values of $h$ the distance between these two fibres can become arbitrary large. Note also that $F_{t}$ depends on $P$ and on $O_{R}$, but not on the location of $R$ on $O_{R}$. The same is true in part (ii) of the following:

Proposition 4.3. (i) For any $h>0$ there exists $k=k(h)>0$, such that any geodesic lift $\gamma=[P, R]$ in $\tilde{M}$ with orbit width $\omega(P, R)<h$ is contained in the horizontal $k$-neighbourhood of the orbits $O_{P} \cup O_{R}$.

(ii) Furthermore, $\gamma$ is $k$-approximated by the union of two vertical segments $\left[P, P_{t}\right] \subset O_{P}$ and $\left[R, R_{t}\right] \subset O_{R}$, where the fibre $F_{t}$ that contains $P_{t}$ and $R_{t}$ is the fibre closest to, say, $P$ where the projection $\gamma_{t} \subset F_{t}$ of $\gamma$ has length $l\left(\gamma_{t}\right) \leq h$.

Proof. We apply Lemma 4.2 (a) to obtain a bound $j=j(k)$ a point $Q \in \gamma$ which lies in the $j$-neighbourhood of the fibre $F_{t}$ described in part (ii) above. The projection $\gamma_{t}$ of $\gamma$ to the fibre $F_{t}$ has length $l\left(\gamma_{t}\right) \leq h$, so that the projection $\gamma_{Q}$ of $\gamma$ to the fibre $F_{Q}$ that contains $Q$ has length $l\left(\gamma_{Q}\right) \leq \lambda^{j} h$. 
Thus we can apply Proposition 4.1, for $d=\lambda^{j} h$, to both subsegments $[P, Q]$ and $[R, Q]$ of $\gamma$ and obtain as direct consequence the desired statements (i) and (ii), for $k=c\left(\lambda^{j} h\right)$.

Proposition 4.4. For any sufficiently large $k>0$ there exists a bound $m=$ $m(k)>0$, such that, for any geodesic lift $\gamma=[P, R]$ in $\tilde{M}$ and any point $Q \in$ $[P, R]$ which lies on the boundary of the horizontal $k$-neighbourhoods of both the orbit $O_{P}$ and the orbit $O_{R}$, the distance from $Q$ to the diagonal projection $\gamma_{\text {diag }}$ of $\gamma$ is smaller or equal to $m$.

Proof. Fix $h>0$. From Proposition 4.3 (i) we know that, if $\omega(P, R)<h$, then $Q$ is contained in the horizontal $k(h)$-neighbourhood of $O_{P} \cup O_{R}$. Thus, for $k>k(h)$ we conclude from the stated hypotheses that $l\left(\gamma_{\text {diag }}\right)=\omega(P, R) \geq h$, while the same hypotheses give $l\left(\gamma_{Q}\right)=2 k$ for the horizontal projection $\gamma_{Q}$ of $\gamma$ which contains $Q$. But since orbits in distinct stable and unstable leaves diverge exponentially in the future and in the past, see section 3 , there is an upper bound $m=m(k)$ to the distance between $Q \in \gamma_{Q}$ and $\gamma_{\text {diag }}$.

\section{$5 \quad$ Geodesic triangles in $\tilde{M}$ are thin}

Proposition 5.1. There exists a constant $b>0$ such any two geodesic lifts $\gamma, \gamma^{\prime}$ in $\tilde{M}$ with same endpoints $A, B$ are b-approximations of each other.

Proof. Fix a constant $\epsilon>2 \delta$ as in Lemma 3.1, let $k(\epsilon)$ be the constant provided by Proposition 4.3, choose $k^{\prime}>k(\epsilon)$, and define $d^{\prime}=3 k^{\prime}$.

If the orbit width $\omega(A, B)$ is smaller than $d^{\prime}$, then both $\gamma$ and $\gamma^{\prime}$ are $k\left(d^{\prime}\right)$ approximated by the same vertical subsegments of $O_{A}$ and $O_{B}$, by Proposition 4.3 , and hence they are $2 k\left(d^{\prime}\right)$-close.

If the orbit width satisfies $\omega(A, B) \geq d^{\prime}$, then, since $k^{\prime}=\frac{d^{\prime}}{3}$, there is a point $Q \in \gamma$ outside of the horizontal $k^{\prime}$-neighbourhood of the endpoint orbits $O_{A}$ and $O_{B}$. Thus there exist points $P, R \in \gamma$ such that $Q \in[P, R] \subset \gamma$, and $Q$ lies on the boundary of the horizontal $k^{\prime}$-neighbourhoods of both orbits $O_{P}$ and $O_{R}$. Thus Proposition 4.4 proves that $Q$ lies in the $m\left(k^{\prime}\right)$-neighbourhood of the diagonal projection $\left[P_{\text {diag }}, R_{\text {diag }}\right]$ at time $t_{\text {diag }}$ of the subsegment $[P, R]$ of $\gamma$. Note also that the geodesic segment $[P, R]$ of $\gamma$ projects to a segment $\left[P_{t}, R_{t}\right]$ in the fibre $F_{t}$ containing $Q$ which has length $d_{\mathcal{F}}^{t}\left(P_{t}, R_{t}\right)=2 k^{\prime}$, so that we deduce $\omega(P, R) \leq 2 k^{\prime}$.

Our choice of the constant $k^{\prime}>k(\epsilon)$ allows us to deduce from Proposition 4.3 (i) that $\omega(P, R) \geq \epsilon$. Furthermore, we know from section 2 that the fibre $F_{\text {diag }}$ which contains $\left[P_{\text {diag }}, R_{\text {diag }}\right]$ is $\delta$-hyperbolic, with respect to the foliation metric $d_{\mathcal{F}}^{t_{\text {diag }}}$. Hence the geodesic $\gamma^{\prime}$ contains points $\hat{P}, \hat{R}$ which are projected by the telescope flow to points $P^{\prime}, R^{\prime} \in F_{\text {diag }}$ in the horizontal $\delta$-neigborhood of $P_{\text {diag }}$ and $R_{\text {diag }}$ respectively. Lemma 3.1 proves that the diagonal time $t^{\prime}$ for $P^{\prime}$ and $R^{\prime}$ satisfies $\left|t_{\text {diag }}-t^{\prime}\right| \leq \sigma$. This gives the possibility to determine upper and lower bounds for $\omega(\hat{P}, \hat{R})$ as follows: $2 k^{\prime}+2 \delta \geq \omega(P, R)+2 \delta \geq d_{\mathcal{F}}^{t_{\text {diag }}}\left(P^{\prime}, R^{\prime}\right) \geq$ $\omega(\hat{P}, \hat{R}) \geq \lambda^{-\sigma} d_{\mathcal{F}}^{t_{\text {diag }}}\left(P^{\prime}, R^{\prime}\right) \geq \lambda^{-\sigma}(\omega(P, R)-2 \delta) \geq \lambda^{-\sigma}(\epsilon-2 \delta)$. We then apply 
Lemma 4.2 (b) with $h_{1}=2 k^{\prime}+2 \delta$ and $h_{0}=\lambda^{-\sigma}(\epsilon-2 \delta)$ and $h=\omega(\hat{P}, \hat{R})$. The last equation implies that in Lemma 4.2 (a) the fibre $F_{t}$ agrees with the diagonal fibre $F_{t^{\prime}}$ for $\hat{P}$ and $\hat{R}$. Thus there is a point $Q^{\prime} \in \gamma^{\prime}$ of distance $\leq j\left(h_{0}, h_{1}\right)$ from the diagonal projection of the segment $[\hat{P}, \hat{R}]$, which itself has distance $\leq \sigma$ from $\left[P_{\text {diag }}, R_{\text {diag }}\right]$, which in turn has length $\leq 2 k^{\prime}$ and contains a point of distance $\leq m\left(k^{\prime}\right)$ from $Q$. This gives the desired uniform upper bound on $d\left(Q, Q^{\prime}\right)$.

Still in the case $\omega(A, B) \geq d^{\prime}$, for any $P \in \gamma$ in the horizontal $k^{\prime}$-neighbourhood of the orbit $O_{A}$ (or of $O_{B}$ ) there is a point $Q$ as above on the boundary of this horizontal neighbourhood, with $P \in[A, Q]$. Then Proposition 4.1 shows that $P$ lies within horizontal distance $c=c\left(k^{\prime}\right)$ from a point on the vertical segment $\left[A, A_{t}\right]$ of $O_{A}$, where $t$ is the time coordinate of $Q$. Now, the point $Q^{\prime} \in \gamma^{\prime}$ constructed above is of bounded distance from $Q$, so that Proposition 4.1 provides a vertical approximation $\left[A, A^{\prime}\right]$ of $\left[A, Q^{\prime}\right]$ with $A^{\prime}$ not far from $A_{t}$. Hence $P$ is close to some point $P^{\prime}$ of $\gamma^{\prime}$.

Proposition 5.2. There exists a constant $k>0$ such that every geodesically lifted triangle $\Delta=[A, B, C]$ in $\tilde{M}$, which projects to a tripod in any fibre, is $k$-thin.

Proof. We proceed in close analogy to the proof (though not to the statement) of Proposition 5.1: Outside a horizontal neighbourhood of the orbit of the triangle vertices and of the tripod centre $O_{Z}$ we conclude as in the above considered case of geodesically lifted digons (or bigons, if you prefer latino-greek imbroglio). Inside these orbit neighbourhoods we use Propositions 4.1 (in the case of large orbit width between $O_{Z}$ and the vertex orbit) and 4.3 (in the case of small orbit width) to approximate the segments of the sides of $\Delta$ by vertical segments on that orbit. The endpoints of these orbit segments are determined by the vertices of $\Delta$ or by the time coordinate of a point $Q$ on the boundary of the above horizontal neighbourhood, if such a point exists (i.e. in the case of large orbit width, where Proposition 4.1 is applied). If for one of the vertices of $\Delta$, say $A$, the orbit width $\omega(A, Z)$ is small, then the corresponding endpoints of the orbit segments on $O_{A}$ and on $O_{Z}$ are determined by the time coordinate of $A_{t} \in O_{A}$ given as the points $P_{t} \in O_{P}$ in Proposition 4.3 (ii). Notice here that we crucially exploit the fact that the fibre $F_{t}$ in Proposition 4.3 (ii) depends on $P$ and on $O_{R}$ but not on the particular location of $R$ on the orbit $O_{R}$, as a priory we do not know whether the two sides $[A, B]$ and $[A, C]$ of $\Delta$ meet the orbit $O_{Z}$ at a close distance.

Thus an endpoint of such a vertical segment is either independent from the side of $\Delta$ which is being approximated, or else it is near to the endpoint of the vertical segment, on the same orbit, which approximates one of the other two sides of $\Delta$. In any case each of the vertical segments is contained in a bounded neighbourhood of the corresponding vertical segment from the other side or from the other two sides of $\Delta$.

Theorem 5.3. There exists a constant $h>0$ such that every geodesic triangle $\Delta=[A, B, C]$ in $\tilde{M}$ is h-thin.

Proof. From Proposition 2.4 we know that there is another triangle $\Delta^{\prime}$ with same endpoints as $\Delta$, but with sides that are only geodesic lifts, such that $\Delta^{\prime}$ is projected by the telescope flow to a tripod, in any fibre $F_{t}$. Now Proposition 5.2 
shows that $\Delta^{\prime}$ is thin, while Proposition 5.1 shows that $\Delta^{\prime}$ is an approximation of $\Delta$, so that $\Delta$ must also be thin.

Theorem 5.3 shows that $\tilde{M}$ is hyperbolic in Gromov's sense. As the action of $\pi_{1} M_{h}$ on $\tilde{M}$ is isometric, free, properly discontinuous and cocompact (see section 3 ), this implies Proposition 0.4 from the introduction as an immediate corollary.

Remark 5.4. An interesting feature of the material presented in this and the previous sections comes from the fact that foliations with a transverse measure determine a dual $\mathbb{R}$-tree. Our proof of Theorem 5.3 generalizes nicely to this more general setting, so that direct extensions of this approach seem natural. This concerns for example certain classes of free group automorphisms, where however a main problem remains to be solved: The action of $F_{n}$ on the product of two $\mathbb{R}$-trees $T_{+} \times T_{-}$is not cocompact and hence one has to find a suitable $F_{n}$-invariant subspace of $T_{+} \times T_{-}$. Recently V. Guirardel has obtained interesting results on this matter, see [18].

\section{Relative hyperbolic mapping tori: The special case}

The goal of this and the next section is to prove Theorem 0.1 from the introduction, or rather, the stronger Theorem 7.1. For this purpose we need to extend techniques (and results) of the previous sections to the case of reducible surface homeomorphisms, replacing "hyperbolicity" of the mapping torus by "relative hyperbolicity". There are two different notions of relative hyperbolicity in the literature, and they are note quite equivalent, though very close. We will give more details below in Remark 7.4. Until then we will use "relative hyperbolic" always in the sense of Farb's paper [9] (called "weakly hyperbolic" in [3]), which we recall now.

Let $G$ be a finitely generated group, and let $\mathcal{U}=\left(U_{1}, \ldots, U_{r}\right)$ be a finite family of subgroups $U_{i}$ of $G$. A Cayley graph $\Gamma_{*}$ of $G$ relative to $\mathcal{U}$ is the Cayley graph $\Gamma$ of $G$ with respect to a finite generating system of $G$, together with extra vertices added, one for each left coset $g U_{i}$, and with extra edges added that connect any such extra vertex to any vertex of $\Gamma$ which is labelled by an element of this coset.

One says that $G$ is relative hyperbolic with respect to $\mathcal{U}$ if a Cayley graph of $G$ relative to $\mathcal{U}$ is a $\delta$-hyperbolic space. It is immediate that any two Cayley graphs of $G$ relative to the same family of subgroups $\mathcal{U}$ are quasi-isometric, so that relative hyperbolicity depends only on $G$ and $\mathcal{U}$. Similarly, replacing any $U_{i}$ of $\mathcal{U}$ by a conjugate $g U_{i} g^{-1}$ or by a finite index subgroup $U_{i}^{\prime} \subset U_{i}$ changes the relative Cayley graph only up to a quasi-isometry.

The canonical action of $G$ on a relative Cayley graph $\Gamma_{*}$ is no longer free, as is the $G$-action on "absolute" Cayley graphs. However, every $G$-orbit in $\Gamma_{*}$ is discrete, and on all but finitely many of them $G$ acts freely. The exceptional orbits $G x_{i}$ are in 1-1 correspondence with the subgroups $U_{i}$ of $\mathcal{U}$, in that $\operatorname{Stab}_{G}\left(x_{i}\right)=$ 
$g_{i} U_{i} g_{i}^{-1}$ for some suitable $g_{i} \in G$. In particular, one easily verifies that the action of $G$ on $\Gamma_{*}$ satisfies the hypotheses of the following proposition, which we will use below. It seems to be (within an $\epsilon$-neighbourhood of) folk knowledge among the experts and can be proved easily by a proper variation of the well known proof in the classical case of empty $\mathcal{U}$, see e.g. [16]:

Proposition 6.1. Let $X$ be a geodesic metric space, and let $G$ be a finitely generated group which acts on $X$ by isometries. Assume that $X / G$ can be covered by finitely many connected sets $B_{i}$ with pairwise connected intersections, and with the property that some (and hence every) connected component $\tilde{B}_{i}$ of the full preimage of any $B_{i}$ in $X$ has finite diameter and is convex. Assume furthermore that there is a lower bound $\epsilon>0$ to the distance between disjoint lifts $g \tilde{B}_{i}$ and $h \tilde{B}_{j}$ for any $g, h \in G$. Let $U_{i} \subset G$ be the stabilizer of $\tilde{B}_{i}$.

Then $X$ is quasi-isometric to the group $G$ relative to the family $\mathcal{U}$ of subgroups $U_{i}$ (i.e. to any Cayley graph of $G$ relative to $\mathcal{U}$ ).

In order to concentrate on the main difficulty in translating our techniques of the previous sections from absolute to relative hyperbolicity we will first consider a special case. In the next section, however, we shall see that this special case is also the main building block for the general relative hyperbolic case.

The special case considered in this section is that of a compact surface $S_{*}$ of negative Euler characteristic with non-empty boundary $\partial S_{*}$. Let $h_{*}$ be a pseudo-Anosov homeomorphism of $S_{*}$. As in Section 1, $S_{*}$ is given a hyperbolic structure, and consequently $\pi_{1} S_{*}$ acts by isometries on the hyperbolic plane, represented by the Poincaré disc $D^{2}$. The action, however, is in the present case not cocompact: The (finitely many) boundary curves of $S_{*}$ are represented by geodesics on the hyperbolic surface $D^{2} / \pi_{1} S$, and without loss of generality we can identify $S_{*}$ with the geodesic compact core of $D^{2} / \pi_{1} S_{*}$, i.e. with the subsurface of $D^{2} / \pi_{1} S_{*}$ obtained by cutting off the infinite-volume funnels along the closed simple geodesics that represent the homotopy class of the boundary curves in $D^{2} / \pi_{1} S_{*}$. We denote by $D_{*}^{2}$ the subdisk of $D^{2}$ which is the full preimage of $S_{*} \subset D^{2} / \pi_{1} S_{*}$ under the covering map $D^{2} \rightarrow D^{2} / \pi_{1} S_{*}$.

As in the closed case, we obtain $\tilde{h}_{*}$-invariant foliations $\mathcal{F}_{u}, \mathcal{F}_{s}$ in $D_{*}^{2}$, with projectively invariant measures $\mu_{u}$ and $\mu_{s}$. There is, however, a substantial difference from the closed case, in that the boundary curves of $S_{*}$ lift to leaves which belong to both foliations $\mathcal{F}_{u}$ and $\mathcal{F}_{s}$. At all other points of $D_{*}^{2}$ the two foliations are transverse as before.

We continue to proceed, nevertheless, just as in the closed case, thus simply ignoring the above "difficulty": We define the mapping telescope $\tilde{M}_{*}$ as universal covering space of the mapping torus $M_{h_{*}}$ and identify it with $D_{*}^{2} \times \mathbb{R}$. The action of $\pi_{1} M_{h_{*}}$ on $\tilde{M}_{*}=D_{*}^{2} \times \mathbb{R}$ is defined precisely as described in section 1 for the closed case. Similarly, we copy from section 1 the notion of fibres and orbits in $\tilde{M}_{*}$, and also the rescaling of the measure on the fibres so that the action of $\pi_{1} M_{h_{*}}$ on $\tilde{M}_{*}$ preserves the transverse measure of any horizontal path in $\tilde{M}_{*}$.

Next we introduce on $D_{*}^{2}$ the foliation distance as described at the beginning of section 2. Note that, because of the above "problem", rather than getting a 
metric, this defines a pseudo-metric space $D_{* \mathcal{F}}^{2}$, where any two distinct points have distance 0 if and only if they belong to the same boundary geodesic of $D_{*}^{2}$. Let $\widehat{D}_{*}^{2}$ denote the canonical metric quotient space of $D_{*}^{2}$. We prove:

Proposition 6.2. The disk $\widehat{D_{* \mathcal{F}}^{2}}$ is a Gromov hyperbolic metric space.

Proof. From Proposition 6.1 we know that $\widehat{D_{* \mathcal{F}}^{2}}$ is quasi-isometric to the free group $\pi_{1} S_{*}$, relative to the set of cyclic subgroups generated by the boundary curves (which are well defined up to conjugacy). Cyclic subgroups are known to be always quasi-convex in any hyperbolic group. By [14] any hyperbolic group $G$ is hyperbolic relative to any quasi-convex subgroup. If $G$ is a free group (as is the fundamental group of a surface with boundary), this follows alternatively from elementary arguments, which also extend directly to the case of more than one relative subgroup, see [13].

As in section 2 one then defines the foliation (pseudo-)metric on each fibre $F_{t}$ of $\tilde{M}_{*}$, and Lemmas 2.2 and 2.3 as well as Proposition 2.4 carry over word by word. Indeed, the same is true for all of sections $3-5$, so that we obtain:

Theorem 6.3. There exists a constant $h>0$ such that every geodesic triangle $\Delta=[A, B, C]$ in $\tilde{M}_{*}$ is h-thin.

Thus we can again apply Proposition 6.1 to obtain:

Corollary 6.4. The group $\pi_{1} M_{h_{*}}$ is hyperbolic relative to the family of cyclic subgroups generated by the boundary components of the surface, i.e. of the fibre.

The reader should note here that this result is not a special case of Farb's relative hyperbolicity result for hyperbolic 3-manifolds with cusps (see [9]), as in the above result the relative subgroups are all infinite cyclic, while Farb considers hyperbolicity relative all of the fundamental group of any cusp neighbourhood, which is isomorphic to $\mathbb{Z} \oplus \mathbb{Z}$.

\section{Relative hyperbolic mapping tori: The gen- eral case}

Let $\mathcal{G}$ be a finite graph of groups with fundamental group $G$, and let $\alpha$ be an automorphism of $G$ which respects the graph of groups structure of $\mathcal{G}$ : There are different equivalent ways to express this, the most elegant saying that the length function $\|\cdot\|_{\mathcal{G}}$, defined by $\mathcal{G}$ on the conjugacy classes of $G$, is invariant under $\alpha$. Equivalently, there is a homeomorphism $H: T \rightarrow T$ of the BassSerre tree $T$ associated to $\mathcal{G}$ which satisfies $\alpha(g) H=H g: T \rightarrow T$ for every $g \in G$. Such an automorphism $\alpha$ induces a graph isomorphism $\alpha_{\Gamma}$ on the graph $\Gamma=T / G$ on which $\mathcal{G}$ is built, and for every vertex $v$ and every edge $e$ of $\Gamma$ group isomorphisms $\alpha_{v}: G_{v} \rightarrow G_{\alpha_{\Gamma}(v)}$ and $\alpha_{e}: G_{e} \rightarrow G_{\alpha_{\Gamma}(e)}$ such that for the terminal vertex $v=\tau(e)$ of any (oriented) edge $e$ and the corresponding group 
monomorphism $f_{e}: G_{e} \rightarrow G_{v}$, given by $\mathcal{G}$, one has $f_{\alpha_{\Gamma}(e)} \alpha_{e}=\alpha_{v} f_{e}$ up to inner automorphisms of $G_{\alpha_{\Gamma}(v)}$. Supposing the existence of such maps $\alpha_{\Gamma}, \alpha_{v}$ and $\alpha_{e}$ gives another equivalent way to describe that $\alpha$ preserves the graph of groups structure of $\mathcal{G}$, see e.g. [6].

Let now $V_{0}$ be an $\alpha_{\Gamma}$-invariant subset of the vertices of $\Gamma$, such that each of the groups $G_{v}$ for $v \in V_{0}$ is the fundamental group of a surface $S_{v}$ with boundary. We assume furthermore that for all edges $e$ with terminal vertex $\tau(e) \in V_{0}$ the edge group $G_{e}$ maps under the edge monomorphism $f_{e}$ into an infinite cyclic subgroup of $G_{\tau(e)}$ that is generated (up to conjugation) by some boundary curve $\partial_{e} S_{\tau(e)}$ of the surface $S_{\tau(e)}$. In particular, these edge groups $G_{e}$ must be infinite cyclic. (One could also allow trivial $G_{e}$, at the expense of minor technical modifications below.)

Finally, for each vertex $v \in V_{0}$ we assume that there is a homeomorphism $h_{v}: S_{v} \rightarrow S_{\alpha_{\Gamma}(v)}$ that induces the group isomorphism $\alpha_{v}: G_{v} \rightarrow G_{\alpha_{\Gamma}(v)}$. We denote by $t_{v}$ the cardinality of the $\alpha_{\Gamma^{-}}$orbit of $v$. We then want to assume that the $t_{v}$-th power of the set of homeomorphisms $h_{v}$ defines on $S_{v}$ a homeomorphism $h_{v}^{\left(t_{v}\right)}=h_{\alpha_{\Gamma}^{t_{v}-1}(v)} h_{\alpha_{\Gamma}^{t_{v}-2}(v)} \ldots h_{\alpha_{\Gamma}(v)} h_{v}$ which is pseudo-Anosov. We call the vertices from $V_{0}$ vertices of type pseudo-Anosov on surface with boundary.

We now define complementary subgroups as follows: For every boundary component $\partial_{i} S_{v}$ of a surface $S_{v}$ with $v \in V_{0}$ one introduces a new vertex $v\left(\partial_{i} S_{v}\right)$ with vertex group isomorphic to $\mathbb{Z}$. The new vertex is connected by a newly introduced edge $e\left(\partial_{i} S_{v}\right)$ to $v$. The new edge group $G_{e\left(\partial_{i} S_{v}\right)}$ is also isomorphic to $\mathbb{Z}$, and on one side of $e\left(\partial_{i} S_{v}\right)$ the edge group monomorphism surjects $G_{e\left(\partial_{i} S_{v}\right)}$ onto $G_{v\left(\partial_{i} S_{v}\right)}$, while on the other side $G_{e\left(\partial_{i} S_{v}\right)}$ is mapped onto the infinite cyclic group $\pi_{1} \partial_{i} S_{v}$. This does not change $\pi_{1} \mathcal{G}$, and now all "old" edges of $\mathcal{G}$ adjacent to $S_{v}$ can be slid over one of the new edges to be attached to one of the new vertices, again without changing $\pi_{1} \mathcal{G}$. We now remove all vertices from $V_{0}$ together with all new edges (but not the new vertices!), and define the subgroups complementary to the vertex subset $V_{0}$ to be the fundamental groups of the obtained connected components of the left over sub-graph of groups. Each component determines a subgroup of $\pi_{1} \mathcal{G}$ that is well defined up to conjugation.

We can now formulate the main result of this paper, in the most general form:

Theorem 7.1. Let $G$ be the fundamental group of a finite graph of groups $\mathcal{G}$, and let $\alpha$ be an automorphism of $G$ which respects the structure of $\mathcal{G}$. Let $V_{0}$ be the set of vertices of type pseudo-Anosov on surface with boundary. Then the semidirect product $G_{\alpha}=G \rtimes_{\alpha} \mathbb{Z}$ is a hyperbolic group relative to the family of subgroups complementary to $V_{0}$.

A direct consequence of this theorem is Theorem 0.3 from the introduction, where the graph of groups structure of $G=\pi_{1} S$ is given by the reduction curves of the Nielsen-Thurston decomposition of the mapping class of $h$.

In order to derive Theorem 0.1, we use Sela's canonical graph of groups decomposition of one-ended hyperbolic groups $G$, as described and specified in [2]. This decomposition is canonical in the sense that it is preserved by any automorphism $\alpha$ of $G$. There are three kind of vertices in this decomposition: For 
torsion-free $G$ the vertex groups are either rigid, infinite cyclic, or quadratically hanging. The edge groups are all infinite cyclic and they contain the infinite cyclic vertex groups. (The latter are only of auxiliary nature, in that one could get rid of them by contracting suitably chosen edges, at the expense of damaging the canonical nature of the decomposition). The quadratically hanging vertex groups are fundamendal groups of surfaces with boundary (and thus free groups), and the adjacent edge groups are given (up to passage to a finite index subgroup) by the boundary components of the surface. Note that for torsion free hyperbolic $G$ the hypotheses "one-ended" and "freely indecomposable non-elementary" are equivalent, by Stalling's celebrated characterisation of groups with infinitely many ends as amalgamated products with finite amalgam [20].

Thus Theorem 7.1 gives directly:

Theorem 7.2. (a) Let $G$ be a freely indecomposable non-elementary torsion-free word-hyperbolic group, and let $\alpha$ be an automorphism of $G$. Assume that in the canonical graph of groups decomposition of $G$ on all of the quadratically hanging vertex groups the restriction of (a power of) $\alpha$ is induced by a pseudo-Anosov homeomorphism. Then the semi-direct product $G \rtimes_{\alpha} \mathbb{Z}$ is hyperbolic relative to the family of subgroups complementary to the set of vertices with quadratically hanging vertex group.

(b) If the above assumption on the quadratically hanging vertex groups is not valid, then the analogous statement holds, provided that the canonical decomposition of $G$ is refined to include as further edges the reduction curves in the NielsenThurston reduction of the induced automorphisms on the quadratically hanging vertex groups. The relative subgroups are then the subgroups complementary to the set of vertices which correspond to those subsurfaces on which $\alpha$ is pseudoAnosov.

Rigid vertex groups can be characterized by the fact that they are nonelementary and have finite outer automorphism group. Thus a power of $\alpha$ fixes every conjugacy class of elements in the vertex groups of the relative part, i.e. of any connected component of the subgraph of groups complementary to the set $V_{0}$ of vertices of pseudo-Anosov type on surface with boundary, in the refined canonical graph of groups $\mathcal{G}$, after having properly introduced the "new" vertices and edges as described before Theorem 7.1. It follows that such an automorphism is a multiple Dehn twist in the sense of [6] on every subgroup of $G$ complementary to the set of vertices from $V_{0}$. Such multiple Dehn twists are well known to have linear or zero growth on all conjugacy classes. Thus the above Theorem 7.2 (together with Proposition 0.4 to include the case where $\mathcal{G}$ consists of a single vertex) implies directly Theorem 0.1 from the introduction.

To prove Theorem 7.1 we construct a topological space $X$ modelled on the graph $\Gamma$ in the usual way, to get $G=\pi_{1} X$. Here each vertex $v$ from $V_{0}$ is represented by the surface $S_{v}$, while all other vertex spaces $X_{v^{\prime}}$ can be taken arbitrary (with $\pi_{1} X_{v^{\prime}}=G_{v^{\prime}}$, of course). The edge spaces $X_{e}$ with $\tau(e) \in V_{0}$ are given by annuli which, in the forward direction of $e$, are attached to a power 
of the boundary curve $\partial_{e} S_{v}$ of the surface $S_{v}$. The universal covering $\tilde{X}$ of $X$, together with the standard action of the fundamental group $G=\pi_{1} X$ by deck transformations, gives then a model for the Bass-Serre tree $T$ associated to $\mathcal{G}$, where each vertex of $T$ is represented by a connected component of the full preimage in $\tilde{X}$ of a vertex space, and conversely. This is quite standard though a little heavy on the notational side, as is typical for Bass-Serre theory.

We now provide each of the surfaces $S_{v}$ with the $h_{v}$-invariant measured foliations and lift the latter to each connected component of the full preimage of $S_{v}$ in $\tilde{X}$. We then continue for each of these connected components precisely as in the special case considered in the previous section, while all the connected components of the full preimage of any other vertex space or of any edge space is given the zero pseudo-metric, thus effectively contracting each such connecting component. Let $\widehat{X}$ denote the obtained metric space.

As in the special case treated in the previous section, we now pass to the mapping telescope $\widehat{M}=\widehat{X} \times \mathbb{R}$, with fibres and orbits as defined before, and with an action of $G \rtimes_{\alpha} \mathbb{Z}$ defined on it so that, with respect to the properly rescaled metric on the fibres and the induced telescope metric on $\widehat{M}$, this action is isometric. Hence, in light of Proposition 6.1, in order to prove Theorem 7.1 it suffices to show the following:

Proposition 7.3. There exists a constant $h \geq 0$ such that all geodesic triangles in the metric space $\widehat{M}$ are $h$-thin.

Proof. We use the tree like structure of $\widehat{M}$, inherited from $T$, to observe that a geodesic triangle is either contained in a vertex space of $\widehat{M}$, in which case we are done, by the special case considered in Theorem 6.3 in the last section. Otherwise, the telescope flow projection of the triangle in any fibre decomposes into finitely many geodesic digons, each contained in a single vertex space, and one geodesic triangle, also contained in a single vertex space. Hence the claim follows again directly from the special case of surfaces with boundary, if we can bound the time coordinate difference which the lifts of the geodesic digons realize on their two sides at the place when they pass from one vertex space to another, i.e. at a "singular orbit" which corresponds to a maximal connected union of edge space and vertex spaces that are not of pseudo-Anosov type on surface with boundary (i.e. a connected component of subspaces that has been contracted above).

To obtain such a bound we first use Proposition 5.1 to reduce the claim to digons with the same telescope flow projections in any fibre. One then uses precisely the same arguments as explained in the proof of Proposition 5.2 for the time coordinate difference of two different geodesic lifts at the tripod centre orbit $O_{Z}$. For digons with endpoints that have small orbit width and which have initial points (i.e. the endpoints closer to the vertices of the given triangle) on the two digon sides already at different time coordinate values, the time coordinate difference at the terminal endpoints can not be larger: This follows from the observation that, in the situation of Proposition 4.3 (ii), moving the point $P$ along its orbit by a distance $\leq t_{0}$, will effect the time coordinate of the fibre $F_{t}$ by at most $t_{0}$, as follows directly from the given definition of $F_{t}$. 
Remark 7.4. There is a stronger version of relative hyperbolicity in the literature, originally proposed by Gromov [17], and later elaborated by Szczepański [21] and Bowditch [3]. It is equivalent (see [21]) to Farb's version of relative hyperbolicity, as used above, plus an aditional property, called "Bounded Coset Penetration property (BCP)" (also due to Farb [9]). The latter, however, implies that the relative subgroups $U_{i}$ must be malnormal in the total group $G$. In our case, this is in general not true, as typically $U_{i}$ is a cyclic summand of a $\mathbb{Z} \oplus \mathbb{Z}$ subgroup in $G$.

Thus, to get the stronger version of relative hyperbolicity for hyperbolic-bycyclic groups, one is forced to increase the relative subgroups, so that with each periodic-up-to-conjugacy element it must contain at least its centralizer, which contains a subgroup isomorphic to $\mathbb{Z} \oplus \mathbb{Z}$. This is done properly by defining new relative subgroups $\widehat{U}_{i}=\left.U_{i} \rtimes_{\alpha^{t}}\right|_{U_{i}} \mathbb{Z}$, where $\alpha^{t}$ is a power of $\alpha$ that fixes every vertex or edge group of $G$ (up to conjugation). It follows then indeed that the group $G_{\alpha}$ from Theorem 7.1 is strongly hyperbolic relative to the family of these subgroups $\widehat{U}_{i}$. To show this one uses again the canonical decomposition of word-hyperbolic groups, where the arguments become actually simpler than in the above treated amalgamation: As the time coordinate must be collapsed as well, there is no need to worry about at which height one enters or leaves a vertex of pseudo-Anosov type on surface with boundary.

To prove the strong relative hyperbolicity of such vertex groups (i.e. the analogue of Corollary 6.4), one has two alternatives: One can either use the telescope metric on the mapping telescope $\tilde{M}_{*}$ as introduced in the last section, modify it properly by contracting each boundary component of $\tilde{M}_{*}$, and then use Lemma 1 of [21] to approximate geodesics in the resulting metric by geodesics with respect to the unmodified telescope metric. One then uses Propositions 4.1 and 4.3 and the existence of a lower bound to the distance between any two distinct boundary leaves to prove Farb's property BCP. Alternatively, one can simply quote [9], thus of course using implicitly Thurston's hyperbolisation of fibered 3-manifolds with pseudo-Anosov monodromy [19].

\section{References}

[1] M. BESTVINA and M. FEIGHN, 'A combination theorem for negatively curved groups', Journal of Differential Geometry 35 (1992), 85-101.

[2] B. BOWDITCH, 'Cut points and canonical splittings of hyperbolic groups', Acta Mathematica 180 (1998), 145-186.

[3] B. BOWDITCH, 'Relatively hyperbolic groups', preprint.

[4] M. BRIDSON and A. HAEFLIGER, Metric spaces of non-positive curvature, Fundamental Principles of Mathematical Science 319, Springer Verlag 1999.

[5] J. CANNON and W.P. THURSTON, 'Group Invariant Peano Curves', preprint. 
[6] M. M. COHEN et M. LUSTIG, 'The conjugacy problem for Dehn twist automorphisms of free groups', Comment. Math. Helv. 74 (1999), 179-200.

[7] D.J. COLLINS and E.C. TURNER, 'Efficient representatives for automorphisms of free products', Michigan Math. Journal 41 (3) (1994), 443-464.

[8] D. COOPER, D.D. LONG and A.W. REID, 'Bundles and finite foliations', Inventiones Mathematicae 118 (1994), 255-283.

[9] B. FARB, 'Relatively hyperbolic groups' Geom. Funct. Anal. 8 (1998), 1-31.

[10] A. FATHI, F. LAUDENBACK and V. POENARU, Travaux de Thurston sur les surfaces, Astérique 66-67, 1979.

[11] D. GABAI, 'The Ubiquitous Nature of Quasi-Minimal Semi-Euclidean Laminations in 3-manifolds', Surveys in Differential Geometry 5, International Press.

[12] D. GABAI and W.H. KAZEZ, 'Group negative curvature for 3-manifolds with genuine laminations', Geometry and Topology 2 (1998), 65-77.

[13] F. GAUTERO, 'Hyperbolicité relative des mapping-torus d'automorphismes des groupes libres', preliminary preprint.

[14] S. GERSTEN, 'Subgroups of word-hyperbolic groups in dimension 2', Journal of the London Mathematical Society 54 (2) (1996), 261-283.

[15] E. GHYS and P. DE LA HARPE, Sur les groupes hyperboliques d'après Mikhael Gromov, Progress in Mathematics 83, Birkhauser, 1990.

[16] E. GHYS and P. DE LA HARPE, 'Infinite groups as geometric objects (after Gromov)', Ergodic theory, symbolic dynamics, and hyperbolic spaces, 299-314, Oxford Univ. Press, 1991.

[17] M. GROMOV, 'Hyperbolic groups', Essays in Group Theory Math. Sci. Res. Inst. Publ. 8, Springer 1987, 75-263.

[18] V. GUIRARDEL, 'Intersection space for group actions on trees', preliminary preprint.

[19] J.P. OTAL, Le théorème d'hyperbolisation pour les variétés fibrées de dimension 3, Astérique 235, 1996.

[20] J. STALLINGS, 'On torsion-free groups with infinitely many ends', Annals of Mathematics 88 (2) (1968), 312-334.

[21] A. SZCZEPAŃSKI, 'Relatively Hyperbolic Groups', Michigan Math. J. 45 (1998), 611-618. 
François Gautero:

Université Blaise Pascal (Clermont-Ferrand II), Campus des Cézeaux, Batiment de Mathématiques, Laboratoire de Mathématiques pures, 63177 Aubière, France Email adress: francois.gautero@math.univ-bpclermont.fr

Martin Lustig:

Mathématiques (LATP), Université d'Aix-Marseille III, Av. Escad. NormandieNiemen, 13397 Marseille 20, France

Email adress: martin.lustig@univ.u-3mrs.fr 